\title{
Cardiovascular risk associated with the use of glitazones, metformin and sufonylureas: meta-analysis of published observational studies
}

Manel Pladevall ${ }^{1} 2^{*}$, Nuria Riera-Guardia' ${ }^{1}$, Andrea V Margulis ${ }^{1}$, Cristina Varas-Lorenzo ${ }^{1}$, Brian Calingaert ${ }^{3}$ and Susana Perez-Gutthann ${ }^{1}$

\begin{abstract}
Background: The results of observational studies evaluating and comparing the cardiovascular safety of glitazones, metformin and sufonylureas are inconsistent.To conduct and evaluate heterogeneity in a meta-analysis of observational studies on the risk of acute myocardial infarction (AMI) or stroke in patients with type 2 diabetes using non-insulin blood glucose-lowering drugs (NIBGLD).

Methods: We systematically identified and reviewed studies evaluating NIBGLD in patients with type 2 diabetes indexed in Medline, Embase, or the Cochrane Library that met prespecified criteria. The quality of included studies was assessed with the RTI item bank. Results were combined using fixed-and random-effects models, and the Higgins $P^{2}$ statistic was used to evaluate heterogeneity. Sensitivity analyses by study quality were conducted.

Results: The summary relative risk (sRR) (95\% Cl) of AMl for rosiglitazone versus pioglitazone was 1.13 (1.04-1.24) $\left[l^{2}=55 \%\right]$. In the sensitivity analysis, heterogeneity was reduced $\left[I^{2}=16 \%\right]$. The sRR $(95 \% \mathrm{Cl})$ of stroke for rosiglitazone versus pioglitazone was $1.18(1.02-1.36)\left[I^{2}=42 \%\right]$. There was strong evidence of heterogeneity related to study quality in the comparisons of rosiglitazone versus metformin and rosiglitazone versus sulfonylureas ( $P \geq 70 \%$ ). The sRR (95\% Cl) of AMl for sulfonylurea versus metformin was $1.24(1.14-1.34)\left[l^{2}=41 \%\right]$ and for pioglitazone versus metformin was 1.02 $(0.75-1.38)\left[l^{2}=17 \%\right]$. Sensitivity analyses decreased heterogeneity in most comparisons.

Conclusion/interpretation: Sulfonylureas increased the risk of AMI by $24 \%$ compared with metformin; an imprecise point estimate indicated no difference in risk of AMI when comparing pioglitazone with metformin. The presence of heterogeneity precluded any conclusions on the other comparisons. The quality assessment was valuable in identifying methodological problems in the individual studies and for analysing potential sources of heterogeneity.
\end{abstract}

Keywords: Blood glucose-lowering drugs, Type 2 diabetes mellitus, Stroke, AMl, Meta-analysis, Cardiovascular safety, Pharmacoepidemiology, Observational studies

\footnotetext{
* Correspondence: mpladevall@rti.org

${ }^{1}$ RTI Health Solutions, Trav. Gracia 56 Atico 1 08006, Barcelona, Spain

${ }^{2}$ The Center for Health Policy and Health Services Research, Henry Ford

Health System, Detroit, Michigan, USA

Full list of author information is available at the end of the article
} 


\section{Background}

Globally, about 1 billion people are overweight or obese, which will cause an increase of epidemic proportions in the number of persons with type 2 diabetes and cardiovascular disease. In 2011, 366 million people had been diagnosed with diabetes, and the number is expected to rise to 552 million by 2030. Most people with diabetes live in the developing world, and this area will see the greatest increase over the next 19 years [1].

Type 2 diabetes mellitus (T2DM) is characterised by high blood glucose levels that cause eye, kidney, and nerve complications and an increased risk for cardiovascular disease. Most of the morbidity and mortality associated with diabetes is due to cardiovascular disease; diabetes is considered by some as a coronary heart disease riskequivalent condition [2]. Although this concept is controversial [3], there is evidence that the risk equivalence might hold at least for women [4].

Controversy has surrounded the cardiovascular safety of some non-insulin blood glucose-lowering drugs (NIBGLD), particularly sulfonylureas and thiazolidinediones. Rosiglitazone was removed from the market in Europe and its use highly restricted in the United States of America (US) after review of safety evaluations from clinical trials suggested that its use increased the incidence of acute myocardial infarction (AMI) [5]. This and the premature termination of the ACCORD trial [6], after observing an increase in cardiovascular mortality in the group treated more aggressively, prompted the US Food and Drug Administration (FDA) to revise its policy for approving new drugs for T2DM; a two-step approach ensuring cardiovascular safety is now required. Evidence has led some to question rosiglitazone's adverse cardiovascular safety profile [7-9]. Using observational data, compared with metformin, sulfonylureas have been associated with a $21 \%$ increase in the risk of hospitalisation for AMI, stroke, or death [10].

Meta-analyses of clinical trials and observational studies reviewing the risk of AMI and/or stroke associated with the use of oral blood glucose-lowering drugs for T2DM have been published [11-18]. However, previous meta-analyses of observational studies have not investigated the heterogeneity present in results and methods of individual studies $[11,15,16]$. Heterogeneity, analysis, important for meta-analyses of observational studies [19], might explain inconsistencies among results of individual studies.

This research was part of the Safety Evaluation of Adverse Reactions in Diabetes (SAFEGUARD) project. This report summarises the results of a systematic review and meta-analysis of published observational studies on the risk of AMI and stroke in patients with T2DM receiving treatment with NIBGLD, analysis of the heterogeneity in study characteristics and results, and evaluation of study quality as an explanatory factor for statistical heterogeneity. We also provide results of drug comparisons not reported in previous meta-analyses. With the recent introduction in clinical practice of new classes of NIBGLD such as dipeptidyl peptidase-4 inhibitors, glucagon-like peptide-1 receptor agonists, and sodium-glucose cotransporter-2 inhibitors, it becomes crucial to evaluate the cardiovascular safety of older NIBGLD (e.g., glitazones, metformin and sufonylureas) before their use in clinical practice may change with the introduction of the newer agents, and newer clinical guidelines.

\section{Methods}

We conducted a systematic literature search in Medline, Embase, and the Cochrane Library. The search was conducted on November 11, 2011 (see Additional file 1), and was limited to observational studies on humans (systematic reviews, meta-analyses, original articles), with no publication date or language restrictions for blood glucose-lowering drugs (except insulin); outcomes were AMI, stroke, and others reported elsewhere with details on the literature search, study selection, and data abstraction [20]. Studies including transient ischaemic attack in the definition of stroke were excluded. Studies including stable angina in the definition of AMI were excluded. We conducted an updated literature search in September 2014, and the impact of additional studies that were identified is reported in the discussion section.

We assessed the quality of each study included in the systematic review using two tools, the Newcastle-Ottawa Scale [21] and the RTI item bank (RTI-IB) on risk of bias and precision [22]. We used a version of the RTI-IB adapted to the research question with 31 items. It was applied independently by two researchers who discussed disagreements until reaching consensus, when necessary. Possible responses for most items denoted high, unclear, or low risk of bias. Additional details on the tool and how the quality assessment was performed are reported elsewhere [23].

Quantitative analysis was conducted using Review Manager software version 5.2.3 [24]. Additional details can be found elsewhere [20]. For each comparison with at least three independent point estimates available, summary relative risks (sRRs) and $95 \%$ CIs for AMI and stroke were estimated using both fixed- and randomeffects models. Forest plots were constructed based on random-effects models, and between-study heterogeneity was assessed by graphical inspection and with the Higgins $I^{2}$ statistic, which describes the percentage of between-study variability in effect estimates attributable to true heterogeneity rather than chance. The Cochran's $X^{2}$ test of homogeneity and $\mathrm{Tau}^{2}$, estimating the between-study variance, are also presented. For studies 
providing estimates for both monotherapy and combined therapy, we selected the combined-therapy estimate because in clinical practice most patients with T2DM require combined therapy and because it would include more patients (more precision). To explore sources of heterogeneity, we implemented subgroup analyses designed a priori [20], followed by sensitivity analyses in which we excluded studies with high/unclear risk of bias for more than $30 \%$ of the items in the RTIIB. An additional sensitivity analysis examined the potential impact of the rosiglitazone controversy on the meta-analysis results by grouping the studies according to whether the study period finished before or on 2007, started after 2007, or included 2007, the year in which the first studies showing an increased cardiovascular risk associated with the use of rosiglitazone were published. Publication bias was examined by visual evaluation of funnel plots. The present report follows the MOOSE (Meta-analysis Of Observational Studies in Epidemiology) checklist [25] (see Additional file 1: Table 10e). The present systematic review of published observational studies does not require ethics approval.

\section{Results and discussion}

\section{Results}

Figure 1 displays the study selection process. From the 44 studies selected for the systematic review, we identified 23 studies evaluating the risk of AMI and 8 studies evaluating the risk of stroke. Of the 23 studies on AMI, 17 contributed to the meta-analysis [26-42], with five drug comparisons: rosiglitazone versus pioglitazone ( $n=11$ studies), rosiglitazone versus metformin $(n=7)$, pioglitazone versus metformin $(n=4)$, rosiglitazone versus sulfonylureas $(n=5)$, and sulfonylureas versus metformin

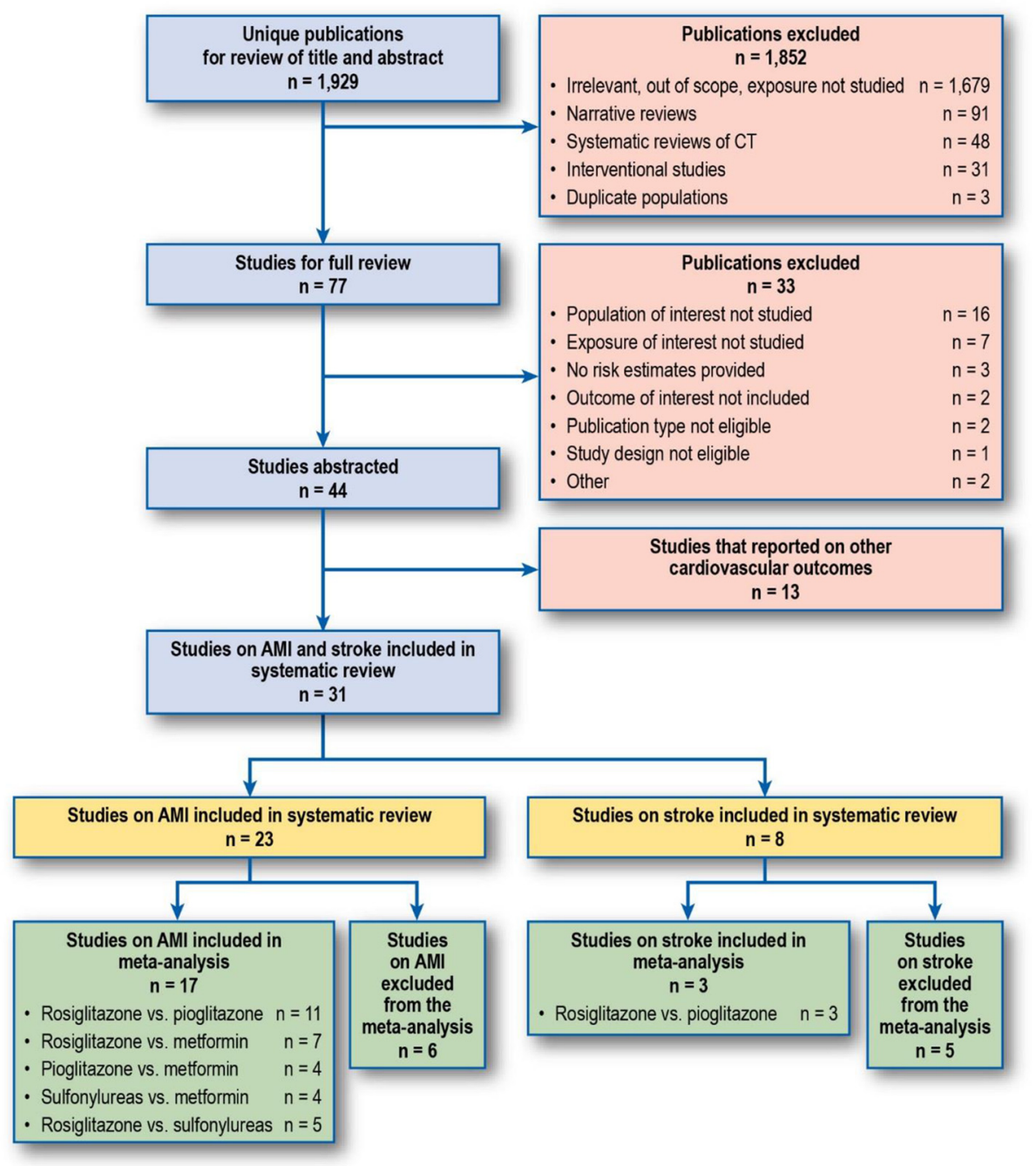

Fig. 1 Flow diagram of study identification and selection process. Note: No additional study was identified by checking reference lists of included studies. Some studies contributed to more than one drug-drug comparison 
$(n=4)$. Of the 8 studies on stroke, 3 contributed to the meta-analysis for the comparison of rosiglitazone versus pioglitazone [26, 30, 41]. Table 1 displays the list of studies excluded from the meta-analyses for AMI and stroke and the reasons for exclusion.

Table 2 displays the main characteristics of the 17 studies evaluating the risk of AMI included in the present meta-analysis. Of the 17 studies, 1 was a case-control study and the rest were cohort studies (3 included a nested case-control analysis). The majority of the studies defined the outcome based on hospitalisations. Most studies included the specific diagnosis code for AMI (i.e., ICD-9 code 410 or ICD-10 codes I21 and I22) in their algorithms to identify cases of AMI; Tzoulaki et al. [38] used Read codes, and Sauer et al. [37] used the Minnesota Heart Study criteria to validate cases of AMI in a field study. Six studies included only first-ever AMI; 11 studies included first-ever AMI plus previous AMI. The number of AMI events in each study ranged from 39 to 15,917 . The majority of studies included a broad age range, while a few were restricted to those aged 65 years or older. The definition of exposure varied across studies: 10 studies included only new users of the drugs studied, and the remaining 7 studies included a combination of new and prevalent users. Thirteen studies included patients from North America, 2 studies included European patients, 1 study was conducted in Israel, and 1 in Taiwan. Most of the US studies used claims databases.

Table 2 displays the main characteristics of the 3 studies evaluating the risk of stroke. All were cohort studies

Table 1 Reasons for exclusion of studies

\begin{tabular}{|c|c|c|}
\hline $\begin{array}{l}\text { Author, } \\
\text { year }\end{array}$ & Endpoint(s) & Reason for exclusion \\
\hline $\begin{array}{l}\text { Chou, } 2011 \\
{[52]}\end{array}$ & Stroke, AMI & $\begin{array}{l}\text { The definitions of both stroke and AMI were } \\
\text { deemed not eligible. This study included } \\
\text { transient ischemic attack in the definition of } \\
\text { stroke and stable angina in the definition of AMI. }\end{array}$ \\
\hline $\begin{array}{l}\text { Azoulay, } \\
2010[58]\end{array}$ & Stroke & \multirow{3}{*}{$\begin{array}{l}\text { Studies excluded due to reference groups } \\
\text { combining several medications (e.g., other } \\
\text { diabetic drugs) }\end{array}$} \\
\hline $\begin{array}{l}\text { Habib, } \\
2009[54]\end{array}$ & Stroke, AMI & \\
\hline $\begin{array}{l}\text { Lipscombe, } \\
2007[57]\end{array}$ & AMl & \\
\hline $\begin{array}{l}\text { Dore, } 2009 \\
{[53]}\end{array}$ & AMI & \multirow{3}{*}{$\begin{array}{l}\text { Studies reporting comparisons for which } \\
\text { inadequate data were available (an inclusion } \\
\text { criterion for this meta-analysis was comparison } \\
\text { with at least three independent point } \\
\text { estimates available) }\end{array}$} \\
\hline $\begin{array}{l}\text { Hsiao, } 2009 \\
{[32]}\end{array}$ & Stroke & \\
\hline $\begin{array}{l}\text { Simpson, } \\
2006[59]\end{array}$ & Stroke & \\
\hline $\begin{array}{l}\text { Horsdal, } \\
2009[55]\end{array}$ & AMI & \multirow[t]{2}{*}{$\begin{array}{l}\text { A more recent study, with updated data, } \\
\text { was available (Horsdal, 2011). }\end{array}$} \\
\hline $\begin{array}{l}\text { Horsdal, } \\
2008[56]\end{array}$ & AMI & \\
\hline
\end{tabular}

comparing rosiglitazone versus pioglitazone [26, 30,41]. The number of events ranged from 32 to 1,869 . Two studies included only elderly subjects that were new users of study medications, while the other study included all subjects aged older than 17 years and both new and prevalent users.

In Figs. 2 and 3, the left panel displays the randomeffects forest plot for the comparisons included in our meta-analysis. Summary results of study quality assessment with the RTI-IB are provided in the right panel. Detailed results of the Newcastle-Ottawa Scale and the RTI-IB for the 31 studies included in the systematic review are in the Additional file 1 . An overview of the experience with and a comparison of the two tools is reported elsewhere [23].

\section{Risk of AMI: rosiglitazone versus pioglitazone}

The overall sRR (95\% CI) was 1.13 (1.04-1.24), and there was evidence of substantial heterogeneity $\left(I^{2}=55 \%\right)$ (Fig. 2). With the RTI-IB, for AMI, 2 studies had a high/ unclear risk of bias for $30 \%$ or more of the items assessed [27, 29]; 2 more studies had a high/unclear risk of bias for 25 to $29 \%$ of the items assessed [26, 40]. For stroke, 1 study had high/unclear risk of bias for $25 \%$ or more of the items assessed [26].

The sensitivity analyses showed that heterogeneity was reduced when restricting the analysis to new users of rosiglitazone and pioglitazone $\left(I^{2}=37 \%\right)$ (Table 3). Even larger reductions in heterogeneity were obtained in the sensitivity analysis excluding the two studies at high/unclear risk of bias for $30 \%$ or more of the items assessed $\left(I^{2}=16 \%\right)$. Heterogeneity was also markedly reduced in the subgroup sensitivity analyses. The sRR did not change much in those analyses and were around 1.10 in most cases, but the $95 \%$ CIs were more conservative; when the two high/unclearbias studies were removed from the first-ever and prior AMI subgroup and the new users subgroup, the randomeffects CIs included the null effect (Table 3).

Of the 11 studies included in the AMI meta-analysis, three reported estimates for monotherapy drug use: one study reported estimates for rosiglitazone and pioglitazone as add-on therapy for metformin users [28], and the rest reported estimates of comparisons in which both rosiglitazone and pioglitazone groups allowed combined treatment with other oral blood glucose-lowering drugs. The monotherapy groups included fewer patients than the combination-therapy groups because very rarely in clinical practice are glitazones prescribed without adjuvant therapy.

\section{Risk of stroke: rosiglitazone versus pioglitazone}

The sRR (95\% CI) was 1.18 (1.02-1.36); there was no strong evidence of heterogeneity $\left(I^{2}=42 \%\right)$. The metaanalysis evaluating the risk of stroke associated with the use of rosiglitazone compared with pioglitazone included 
Table 2 Main characteristics of studies included in the meta-analysis

\begin{tabular}{|c|c|c|c|c|c|c|c|}
\hline $\begin{array}{l}\text { Reference, source } \\
\text { population, study } \\
\text { period }\end{array}$ & $\begin{array}{l}\text { Study design, population } \\
\text { size, age }\end{array}$ & $\begin{array}{l}\text { Diabetes type } 2 \\
\text { population } \\
\text { definition }\end{array}$ & $\begin{array}{l}\text { Study endpoints } \\
\text { (number of cases) }\end{array}$ & Case validation & Exposure assessment & $\begin{array}{l}\text { Exposure } \\
\text { recency }\end{array}$ & $\begin{array}{l}\text { Exposure group(s) vs. reference group (n)A: } \\
\text { Comparison(s) contributing to meta-analysisB: } \\
\text { Other comparison(s) }\end{array}$ \\
\hline \multicolumn{8}{|c|}{ Studies included in both meta-analysis endpoints, AMI and stroke } \\
\hline $\begin{array}{l}\text { Bilik, } 2010 \\
\text { [26]TRIAD study } \\
\text { group USA2000- } \\
2003\end{array}$ & $\begin{array}{l}\text { Cohort } N=2,382 \mathrm{Age} \geq \\
18 \text { years (patients taking } \\
\text { only insulin and aged } \\
\text { younger than } 30 \text { years } \\
\text { were excluded) }\end{array}$ & $\begin{array}{l}\text { First prescription } \\
\text { for glitazones. } \\
\text { Patients filling } \\
\text { prescriptions for } \\
\text { more than one } \\
\text { TZD were } \\
\text { excluded }\end{array}$ & $\begin{array}{l}\text { Non-fatal AMI (ICD-9: } \\
410)(N=39) \text { Non- } \\
\text { fatal stroke (ICD-9: } \\
\text { 431, 433, 434) }(N= \\
32)\end{array}$ & None & $\begin{array}{l}\text { Prevalent and new } \\
\text { usersDispensed } \\
\text { prescriptions }\end{array}$ & $\begin{array}{l}\text { Current, } \\
\text { continuous } \\
\text { use until } \\
90 \text { days after } \\
\text { the supply } \\
\text { date of their } \\
\text { most recently } \\
\text { filled } \\
\text { prescription } \\
\text { duration }\end{array}$ & $\begin{array}{l}\text { A: Rosiglitazone } \\
(n=773) \text { vs. pioglitazone } \\
(n=711)\end{array}$ \\
\hline $\begin{array}{l}\text { Graham, } 2010 \\
\text { [30]Medicare, } \\
\text { USA2006-2009 }\end{array}$ & $\begin{array}{l}\text { Cohort227,571Age } \geq \\
65 \text { years }\end{array}$ & $\begin{array}{l}\text { First prescriptions } \\
\text { for glitazones }\end{array}$ & $\begin{array}{l}\text { Hospitalisation for } \\
\text { fatal and non-fatal } \\
\text { AMI/ACS/SCHD(ICD- } \\
9: 410)(N= \\
1,746) \text { Hospitalisation } \\
\text { for fatal and non- } \\
\text { fatal acute stroke, is- } \\
\text { chaemic or haemor- } \\
\text { rhagic(ICD-9: } 430 \text {, } \\
431,433 . \times 1,434 . \times 1 \\
\text { and } 436)(N=1,052)\end{array}$ & $\begin{array}{l}\text { ExternalPPV for } \\
\text { AMI: } 89 \% \text { - } \\
97 \% \text { PPV for } \\
\text { stroke: } 92 \%- \\
100 \%\end{array}$ & $\begin{array}{l}\text { New usersDispensed } \\
\text { prescriptions }\end{array}$ & $\begin{array}{l}\text { Current } \\
\text { continuous } \\
\text { use, including } \\
\text { a gap of no } \\
\text { more than } \\
7 \text { days }\end{array}$ & $\begin{array}{l}\text { A: Rosiglitazone } \\
(n=67,593) \text { vs. } \\
\text { pioglitazone } \\
(n=159,978)\end{array}$ \\
\hline $\begin{array}{l}\text { Winkelmayer, } 2008 \\
\text { [41]Medicare, New } \\
\text { Jersey, USA1999- } \\
2005\end{array}$ & $\begin{array}{l}\text { Cohort28,361Age }> \\
65 \text { years }\end{array}$ & $\begin{array}{l}\text { First prescription } \\
\text { for a glitazone, } \\
\text { regardless of } \\
\text { previous } \\
\text { treatment with } \\
\text { other diabetic } \\
\text { drug(s) }\end{array}$ & $\begin{array}{l}\text { Ever and first } \\
\text { everFatal and non- } \\
\text { fatal AMI (ICD-9: not } \\
\text { reported })(n= \\
\text { 737)Hospitalisation } \\
\text { for a non-fatal } \\
\text { stroke: ischaemic or } \\
\text { haemorrhagic(ICD-9: } \\
433,434,436)(N= \\
1,869)\end{array}$ & $\begin{array}{l}\text { ExternalPPV for } \\
\text { AMI: } 94 \% \text { PPV for } \\
\text { stroke: } 96 \%\end{array}$ & $\begin{array}{l}\text { New usersDispensed } \\
\text { prescriptions }\end{array}$ & $\begin{array}{l}\text { Current, } \\
\text { continuous } \\
\text { use until } \\
60 \text { days after } \\
\text { the end of } \\
\text { supply date of } \\
\text { their most } \\
\text { recently filled } \\
\text { prescription } \\
\text { duration or } \\
\text { until } \\
\text { switching to } \\
\text { other TZD }\end{array}$ & $\begin{array}{l}\text { A: Rosiglitazone } \\
(n=14,101) \text { vs. } \\
\text { pioglitazone } \\
(n=14,260)\end{array}$ \\
\hline \multicolumn{8}{|c|}{ Studies included only in the meta-analysis of AMI } \\
\hline $\begin{array}{l}\text { Horsdal, } 2011 \\
\text { [31]Danish } \\
\text { National } \\
\text { Registries1996- } \\
2004\end{array}$ & $\begin{array}{l}\text { Nested case-control } N= \\
101,313>30 \text { years }\end{array}$ & $\begin{array}{l}\text { Subjects were } \\
\text { classified as } \\
\text { having T1DM and } \\
\text { excluded if they } \\
\text { were aged } \\
\text { younger than } \\
30 \text { years at the } \\
\text { time of their first }\end{array}$ & $\begin{array}{l}\text { Hospitalisation for } \\
\text { AMI (codes not } \\
\text { reported) }(N=10,616)\end{array}$ & External & $\begin{array}{l}\text { Prevalent and new } \\
\text { usersDispensed } \\
\text { prescriptions }\end{array}$ & $\begin{array}{l}\text { At least one } \\
\text { prescription of } \\
\text { study drug } \\
\text { within } \\
90 \text { days } \\
\text { before } \\
\text { hospitalisation }\end{array}$ & $\begin{array}{l}\text { A: Sulfonylurea monotherapy }(n=26,778) \text { vs. } \\
\text { metformin monotherapy }(n=5,927) B \text { : } \\
\text { Sulfonylurea monotherapy }(n=26,778) \text { vs. } \\
\text { any combination }(n=12,425) \text {; metformin } \\
\text { monotherapy }(n=5,927) \text { vs. individual } \\
\text { sulfonylurea monotherapy }(n=26,778)\end{array}$ \\
\hline
\end{tabular}


Table 2 Main characteristics of studies included in the meta-analysis (Continued)

\begin{tabular}{|c|c|c|c|c|c|c|c|}
\hline & & $\begin{array}{l}\text { diagnosis and had } \\
\text { never received a } \\
\text { prescription for an } \\
\text { oral glucose- } \\
\text { lowering drug. } \\
\text { Subjects with } \\
\text { T2DM were those } \\
\text { with codes for } \\
\text { diabetes mellitus } \\
\text { who had not } \\
\text { received } \\
\text { pharmacotherapy, } \\
\text { had received } \\
\text { prescriptions for } \\
\text { oral glucose- } \\
\text { lowering drugs, or } \\
\text { were aged older } \\
\text { than } 30 \text { years } \\
\text { when they had } \\
\text { their first } \\
\text { diagnostic code or } \\
\text { prescription. }\end{array}$ & & & & & \\
\hline $\begin{array}{l}\text { Loebstein, } 2011 \\
\text { [35]Maccabi } \\
\text { Healthcare } \\
\text { Services, } \\
\text { Israel2000-2007 }\end{array}$ & $\begin{array}{l}\text { Cohort } N=15,436 \mathrm{Age}, \\
\text { mean (SD): } 59.1(11.4) \\
\text { years }\end{array}$ & $\begin{array}{l}\text { Subjects in the } \\
\text { Maccabi diabetes } \\
\text { registry with } \\
\text { prescriptions for } \\
\text { rosiglitazone or } \\
\text { metformin for at } \\
\text { least } 6 \text { months }\end{array}$ & $\begin{array}{l}\text { Hospitalisation for } \\
\text { AMI(ICD-9 and Y } \\
\text { codes 410XX, } \\
\text { Y139XX and } \\
\text { Y225XX)(N }=645)\end{array}$ & None & $\begin{array}{l}\text { Prevalent and new } \\
\text { usersDispensed } \\
\text { prescriptions }\end{array}$ & $\begin{array}{l}\text { Current, } \\
\text { continuous } \\
\text { use within } \\
\text { study period } \\
\text { with gaps not } \\
\text { longer than } \\
3 \text { months }\end{array}$ & $\begin{array}{l}\text { A: Rosiglitazone monotherapy } \\
(n=745) \text { or in combination with } \\
\text { metformin ( } n=2,753) \text { vs. metformin } \\
\text { monotherapy ( } n=11,938) \text { (Formulary } \\
\text { restriction allowed to use rosiglitazone only if } \\
\text { inadequate control from SU, } \\
\text { metformin, or both) }\end{array}$ \\
\hline $\begin{array}{l}\text { Brownstein, } 2010 \\
\text { [27]Partners } \\
\text { Healthcare System: } \\
\text { Research Patient } \\
\text { Data Registry, } \\
\text { USA2000-2006 }\end{array}$ & $\begin{array}{l}\text { Cohort } N=26,375 \mathrm{Age} \geq \\
18 \text { years }\end{array}$ & $\begin{array}{l}\text { ICD-9: } 250 . X X \text { or } \\
\text { hemoglobin A1C } \\
\text { of at least } 6.0 \% \\
\text { and at least one } \\
\text { record of } \\
\text { prescription of an } \\
\text { oral diabetes } \\
\text { medication as an } \\
\text { outpatient or } \\
\text { dispensing as an } \\
\text { inpatient }\end{array}$ & $\begin{array}{l}\text { Hospitalisation for } \\
\text { AMI (ICD-9: 410)(N= } \\
1,343)\end{array}$ & $\begin{array}{l}\text { ExternalPPV: } \\
92 \%-94 \%\end{array}$ & $\begin{array}{l}\text { Prevalent and new } \\
\text { usersPrescriptions } \\
\text { issued and dispensed }\end{array}$ & $\begin{array}{l}\text { Current, } \\
\text { continuous } \\
\text { use within } \\
\text { study period } \\
\text { with gaps not } \\
\text { longer than } \\
6 \text { months }\end{array}$ & $\begin{array}{l}\text { A: Rosiglitazone monotherapy } \\
(n=1,879) \text { vs. pioglitazone } \\
\text { monotherapy }(n=806) \text { or } \\
\text { metformin monotherapy }(n=12,490) \\
\text { or sulfonylurea monotherapy }(n=11,200)\end{array}$ \\
\hline $\begin{array}{l}\text { Wertz, } 2010 \\
\text { [40]HealthCore } \\
\text { Integrated } \\
\text { Research } \\
\text { Database, } \\
\text { USA2001-2005 }\end{array}$ & $\begin{array}{l}\text { Cohort } N=36,628 \mathrm{Age} \geq \\
18 \text { years }\end{array}$ & $\begin{array}{l}\text { First prescription } \\
\text { for glitazones }\end{array}$ & $\begin{array}{l}\text { Hospitalisation for } \\
\text { AMI (ED visits } \\
\text { included) (ICD-9 } \\
410 . x x)(n=217)\end{array}$ & None & $\begin{array}{l}\text { New usersDispensed } \\
\text { prescriptions }\end{array}$ & $\begin{array}{l}\text { Current use if } \\
\text { refill occurred } \\
<1.5 \text { times } \\
\text { the days' } \\
\text { supply of the } \\
\text { preceding } \\
\text { claim for TZD }\end{array}$ & $\begin{array}{l}\text { A: Rosiglitazone } \\
(n=14,469) \text { vs. } \\
\text { pioglitazone } \\
(n=14,469)\end{array}$ \\
\hline $\begin{array}{l}\text { Dormuth, } 2009 \\
\text { [28] British }\end{array}$ & & $\begin{array}{l}\text { Subjects with a } \\
\text { pharmacy }\end{array}$ & $\begin{array}{l}\text { Hospitalisations for } \\
\text { fatal and non-fatal }\end{array}$ & None & $\begin{array}{l}\text { New usersDispensed } \\
\text { prescriptions }\end{array}$ & $\begin{array}{l}\text { Current use } \\
\text { within }\end{array}$ & $\begin{array}{l}\text { A: Rosiglitazone } \\
(n=462) \text { vs. pioglitazone }\end{array}$ \\
\hline
\end{tabular}


Table 2 Main characteristics of studies included in the meta-analysis (Continued)

\begin{tabular}{|c|c|c|c|c|c|c|c|}
\hline $\begin{array}{l}\text { Columbia Health } \\
\text { databases, } \\
\text { Canada1997-2007 }\end{array}$ & $\begin{array}{l}\text { Nested case-control } N= \\
11,147 \text { Age, mean }(S D): 70 \\
(12) \text { years }\end{array}$ & $\begin{array}{l}\text { dispensing for } \\
\text { metformin, } \\
\text { without a } \\
\text { dispensing of } \\
\text { metformin, other } \\
\text { antidiabetic } \\
\text { medication, or } \\
\text { insulin in the } \\
\text { previous } 365 \text { days }\end{array}$ & $\begin{array}{l}\text { AMI (ICD-9: 410) }(N= \\
2,244)\end{array}$ & & & $\begin{array}{l}90 \text { days of the } \\
\text { index date }\end{array}$ & $\begin{array}{l}\text { ( } n=235) \text { Rosiglitazone } \\
(n=462) \text { vs. metformin } \\
(n=10,685) \text { Rosiglitazone } \\
(n=462) \text { vs. sulfonylurea } \\
(n=1,612) \text { Pioglitazone } \\
(n=235) \text { vs. metformin } \\
(n=10,912) \text { Sulfonylurea } \\
(n=1,612) \text { vs. metformin } \\
(n=9,535)\end{array}$ \\
\hline $\begin{array}{l}\text { Hsiao, } 2009 \\
\text { [32]Taiwan Health } \\
\text { Insurance } \\
\text { Database2001- } \\
2005\end{array}$ & $\begin{array}{l}\text { Cohort } N=473,483 \text { Age, } \\
\text { not reported }\end{array}$ & $\begin{array}{l}\text { Subjects with their } \\
\text { first ambulatory } \\
\text { visit with ICD-9- } \\
\text { CM code 250.xx } \\
\text { who were pre- } \\
\text { scribed oral blood } \\
\text { glucose-lowering } \\
\text { agents at least } \\
\text { three times. Sub- } \\
\text { jects were ex- } \\
\text { cluded if they had } \\
\text { T1DM (ICD-9-CM } \\
\text { codes 250.x1) or if } \\
\text { they had been } \\
\text { prescribed only in- } \\
\text { sulin during the } \\
\text { study period. }\end{array}$ & $\begin{array}{l}\text { Fatal and non-fatal } \\
\text { hospitalisation for } \\
\text { AMl (ICD-9: } 410 . x x \\
\text { and 411.xx)(N= } \\
15,917)\end{array}$ & None & $\begin{array}{l}\text { New usersDispensed } \\
\text { prescriptions }\end{array}$ & $\begin{array}{l}\text { Current, } \\
\text { continuous } \\
\text { use during } \\
\text { study period }\end{array}$ & $\begin{array}{l}\text { A: Pioglitazone monotherapy } \\
(n=495) \text { or rosiglitazone } \\
\text { monotherapy }(n=2,093) \text { vs. } \\
\text { metformin-based therapy } \\
(n=46,444) \text { and vs. SU-based } \\
\text { therapy }(n=97,651) B \text { : } \\
\text { Pioglitazone }+ \text { SU + metformin } \\
(n=9,510) \text { vs. Rosiglitazone + } \\
\text { SU + metformin } \\
(n=39,962) \text { Pioglitazone + } \\
\text { metformin ( } n=774) \text { vs. } \\
\text { rosiglitazone + metformin } \\
(n=2,408) \text { Pioglitazone }+S U \\
(n=1,231) \text { vs. rosiglitazone } \\
+S U(n=5,141)\end{array}$ \\
\hline $\begin{array}{l}\text { Juurlink, } 2009 \\
\text { [33]Ontario } \\
\text { diabetes database, } \\
\text { Canada2002-2008 }\end{array}$ & $\begin{array}{l}\text { Cohort } N=39,736 \text { Age } \geq \\
66 \text { years }\end{array}$ & $\begin{array}{l}\text { First prescription } \\
\text { for a glitazone }\end{array}$ & $\begin{array}{l}\text { Hospitalisation for } \\
\text { AMI (ICD-10: I20, I21, } \\
\text { I22)(N=698) }\end{array}$ & ExternalPPV $\approx 90 \%$ & $\begin{array}{l}\text { New users } \\
\text { prescriptionDispensed } \\
\text { prescriptions }\end{array}$ & $\begin{array}{l}\text { Current use if } \\
\text { refill occurred } \\
<1.5 \text { times } \\
\text { the days' } \\
\text { supply of the } \\
\text { preceding } \\
\text { TZD claim }\end{array}$ & $\begin{array}{l}\text { A: Rosiglitazone }(n=22,785) \\
\text { vs. pioglitazone }(n=16,951)\end{array}$ \\
\hline \multirow[t]{2}{*}{$\begin{array}{l}\text { Tzoulaki, } 2009 \\
\text { [38]GPRD, United } \\
\text { Kingdom1990- } \\
2005\end{array}$} & $\begin{array}{l}\text { Cohort } N=91,521 \text { Age } 35- \\
90 \text { years }\end{array}$ & $\begin{array}{l}\text { One episode of } \\
\text { care associated } \\
\text { with a clinical or } \\
\text { referral event for } \\
\text { diabetes and } \\
\text { prescriptions for } \\
\text { oral blood } \\
\text { glucose-lowering } \\
\text { treatment }\end{array}$ & $\begin{array}{l}\text { First ever diagnosis } \\
\text { of AMl according to } \\
\text { Read codes ( } N= \\
3,588)\end{array}$ & $\begin{array}{l}\text { ExternalConfirmed } \\
90 \% \text { of AMl } \\
\text { diagnoses }\end{array}$ & $\begin{array}{l}\text { Prevalent and new } \\
\text { usersPrescriptions } \\
\text { issued }\end{array}$ & $\begin{array}{l}\text { Current, } \\
\text { continuous } \\
\text { intervals of } \\
\text { use within the } \\
\text { study period }\end{array}$ & $\begin{array}{l}\text { A: First-generation SU } \\
\text { monotherapy }(n=6,053) \\
\text { or second-generation SU } \\
\text { monotherapy ( } n=58,095) \\
\text { or rosiglitazone monotherapy } \\
(n=8,442) \text { and combination } \\
\text { therapy }(n=9,640) \text { or } \\
\text { pioglitazone including } \\
\text { monotherapy and combination } \\
\text { therapy }(n=3,816) \text { vs. metformin } \\
\text { ( } n=68,181) \text { B: Glibenclamide or } \\
\text { gliclazide or glimepiride or } \\
\text { glipizide or gliquidone vs. } \\
\text { metformin }(n=68,181)\end{array}$ \\
\hline & $\begin{array}{l}\text { Cohort } N=95,002 \text { Age } \geq \\
18 \text { years }\end{array}$ & $\begin{array}{l}\text { Initiators of } \\
\text { glitazones }\end{array}$ & $\begin{array}{l}\text { Hospitalisations for } \\
\text { fatal and non-fatal }\end{array}$ & External & $\begin{array}{l}\text { New usersDispensed } \\
\text { prescriptions }\end{array}$ & $\begin{array}{l}\text { Current, use } \\
\text { at index date }\end{array}$ & \\
\hline
\end{tabular}


Table 2 Main characteristics of studies included in the meta-analysis (Continued)

\begin{tabular}{|c|c|c|c|c|c|c|c|}
\hline $\begin{array}{l}\text { Ziyadeh, } 2009 \\
{[42] \text { i3, USA2000- }} \\
2007\end{array}$ & & & $\begin{array}{l}\text { AMI (ICD-9: } \\
410 . x x)(N=460)\end{array}$ & & & & $\begin{array}{l}\text { A: Rosiglitazone monotherapy } \\
(n=47,501) \text { vs. pioglitazone } \\
\text { monotherapy ( } n=47,501)\end{array}$ \\
\hline $\begin{array}{l}\text { Koro, } 2008 \\
\text { [34]Integrated } \\
\text { HealthCore } \\
\text { Information } \\
\text { Services, } \\
\text { USA1999-2006 }\end{array}$ & $\begin{array}{l}\text { Nested case-control } \\
N=891,901 \text { Age } \geq 30 \text { years }\end{array}$ & $\begin{array}{l}\text { Subjects with a } \\
\text { diagnosis of type } \\
2 \text { diabetes and at } \\
\text { least one } \\
\text { prescription claim } \\
\text { for an antidiabetic } \\
\text { agent during their } \\
\text { follow-up time } \\
\text { available in the } \\
\text { database }\end{array}$ & $\begin{array}{l}\text { First-ever } \\
\text { hospitalisation for } \\
\text { AMI (ICD-9: } \\
410 . x \mathrm{x})(N=9,870)\end{array}$ & None & $\begin{array}{l}\text { Prevalent and new } \\
\text { usersDispensed } \\
\text { prescriptions }\end{array}$ & $\begin{array}{l}\text { Current use, a } \\
\text { prescription in } \\
\text { the last } \\
3 \text { months } \\
\text { prior to index } \\
\text { date }\end{array}$ & $\begin{array}{l}\text { A: Rosiglitazone }(n=3,839) \\
\text { vs. pioglitazone }(n=3,343)\end{array}$ \\
\hline $\begin{array}{l}\text { Walker, } 2008 \\
\text { [39]Pharmetrics } \\
\text { integrated } \\
\text { outcomes } \\
\text { databaseUSA2000- } \\
2007\end{array}$ & $\begin{array}{l}\text { Cohort } \approx 543,000 \text { Age } \geq \\
18 \text { years }\end{array}$ & $\begin{array}{l}\text { Subjects were } \\
\text { users of } \\
\text { rosiglitazone, } \\
\text { pioglitazone, } \\
\text { metformin, or a } \\
\text { sulfonylurea }\end{array}$ & $\begin{array}{l}\text { Hospitalisation for } \\
\text { fatal and non-fatal } \\
\text { AMI (no codes } \\
\text { reported) }(N=502)\end{array}$ & None & $\begin{array}{l}\text { New usersDispensed } \\
\text { prescriptions }\end{array}$ & $\begin{array}{l}\text { Current, use } \\
\text { at index date }\end{array}$ & $\begin{array}{l}\text { A: Rosiglitazone monotherapy } \\
\text { ( } n=12,440) \text { vs. pioglitazone } \\
\text { monotherapy ( } n=16,302) ; \\
\text { rosiglitazone monotherapy } \\
\text { ( } n=12,440) \text { or pioglitazone } \\
\text { monotherapy }(16,302) \text { vs. } \\
\text { metformin }(n=131,075) ; \\
\text { rosiglitazone monotherapy } \\
\text { ( } n=12,440) \text { vs. sulfonylurea } \\
\text { monotherapy ( } n=48,376)\end{array}$ \\
\hline $\begin{array}{l}\text { Gerrits, } 2007 \\
\text { [29]Ingenix } \\
\text { Research } \\
\text { Database, } \\
\text { USA2003-2006 }\end{array}$ & $\begin{array}{l}\text { Cohort } N=29,911 \text { Age } \geq \\
45 \text { years }\end{array}$ & $\begin{array}{l}\text { Subjects with ICD- } \\
9 \text { code of } 250 . x x \\
\text { and a dispensing } \\
\text { of pioglitazone or } \\
\text { rosiglitazone }\end{array}$ & $\begin{array}{l}\text { Hospitalisation for } \\
\text { AMI (ICD-9: } \\
410 . x \mathrm{x})(N=375)\end{array}$ & $\begin{array}{l}\text { External;PPV } \geq \\
95 \%\end{array}$ & $\begin{array}{l}\text { New usersDispensed } \\
\text { prescriptions }\end{array}$ & $\begin{array}{l}\text { Exposure to } \\
\text { pioglitazone } \\
\text { and } \\
\text { rosiglitazone } \\
\text { was treated as } \\
\text { a } \\
\text { unidirectional } \\
\text { time-varying } \\
\text { covariate; that } \\
\text { is, once a pa- } \\
\text { tient met the } \\
\text { exposure def- } \\
\text { inition, the } \\
\text { patient was } \\
\text { considered } \\
\text { exposed from } \\
\text { that point for- } \\
\text { ward, even if } \\
\text { the index } \\
\text { drug was } \\
\text { discontinued }\end{array}$ & $\begin{array}{l}\text { A: Rosiglitazone }(n=15,104) \\
\text { vs. pioglitazone }(n=14,807)\end{array}$ \\
\hline $\begin{array}{l}\text { McAfee, } 2007 \\
\text { [36]Ingenix } \\
\text { Research } \\
\text { Database, } \\
\text { USA2000-2004 }\end{array}$ & $\begin{array}{l}\text { Cohort } N=33,363 \text { Age } \geq \\
18 \text { years }\end{array}$ & $\begin{array}{l}\text { Initiators of } \\
\text { rosiglitazone, } \\
\text { metformin, or a } \\
\text { sulfonylurea }\end{array}$ & $\begin{array}{l}\text { Hospitalisation for } \\
\text { AMI (ICD-9: } \\
410 . x \mathrm{x})(N=226)\end{array}$ & External & $\begin{array}{l}\text { New usersDispensed } \\
\text { prescriptions }\end{array}$ & $\begin{array}{l}\text { Current use } \\
\text { during study } \\
\text { period. } \\
\text { Dispensing of } \\
\text { a different }\end{array}$ & $\begin{array}{l}\text { A: Rosiglitazone monotherapy } \\
\text { ( } n=8,977) \text { vs. metformin } \\
\text { monotherapy }(n=8,977) \\
\text { or sulfonylureas monotherapy } \\
(n=8,977) \text { B:Rosiglitazone + }\end{array}$ \\
\hline
\end{tabular}


Table 2 Main characteristics of studies included in the meta-analysis (Continued)

\begin{tabular}{|c|c|c|c|c|c|}
\hline $\begin{array}{l}\text { Sauer, } 2006 \\
\text { [37]Philadelphia } \\
\text { Metropolitan area, } \\
\text { USA1998-2002 }\end{array}$ & $\begin{array}{l}\text { Case-control (field } \\
\text { study)Controls were } \\
\text { community controls } \\
\text { selected using random } \\
\text { digit dialing } N=764 \text { Age } \\
40-75 \text { years }\end{array}$ & $\begin{array}{l}\text { Subjects with } \\
\text { T2DM treated } \\
\text { with antidiabetic } \\
\text { drugs or diet only }\end{array}$ & $\begin{array}{l}\text { First-ever AMl } \\
\text { (identified using } \\
\text { medical records)(N } \\
=113)\end{array}$ & $\begin{array}{l}\text { AMI validated by } \\
\text { Minnesota Heart } \\
\text { Survey criteria }\end{array}$ & $\begin{array}{l}\text { Prevalent and new } \\
\text { usersInterviews }\end{array}$ \\
\hline
\end{tabular}

study drug or

insulin for the

monotherapy

group (at

which time

the subjec

became

eligible for a

different study

cohort);

cessation of

study drug

use alone was

not sufficient

to end follow-

up

Current, use

in the 7 days

before index

date

A: Sulfonylurea monotherapy

$(n=158)$ vs. metformin

monotherapy $(n=125)$ B:

Sulfonylureas $(n=158)$ or

metformin (125) vs.

thiazolidinedione $(n=26)$

Sulfonylureas $(n=158)$ vs.

thiazolidinediones + sulfonylureas

$(n=27)$ Sulfonylureas $(n=158)$ vs

metformin + sulfonylureas

$(n=102)$ Metformin $(n=125)$ vs.

thiazolidinediones + metformin

$(n=21)$

ACS acute coronary syndrome, AMI acute myocardial infarction, ED emergency department, GPRD General Practice Research Database (now the Clinical Practice Research Datalink [CPRD]), hemoglobin A1C glycated hemoglobin, ICD-9 international classification of diseases, 9th revision, ICD-9-CM, International Classification of Diseases, 9th Revision, Clinical Modification; ICD-10, International Statistical Classification of Diseases and Related Health Problems, 10th Revision; PPV, positive predictive value; SCHD, serious coronary heart disease; SD, standard deviation; SU, sulphonylurea(s); T1DM, type 1 diabetes mellitus; T2DM type 2 diabetes mellitus, TZD thiazolidinedione(s), USA United States of America

Note: When it is not indicated that the endpoint is the first ever identified, the study included patients with and without prior history of the study endpoint 


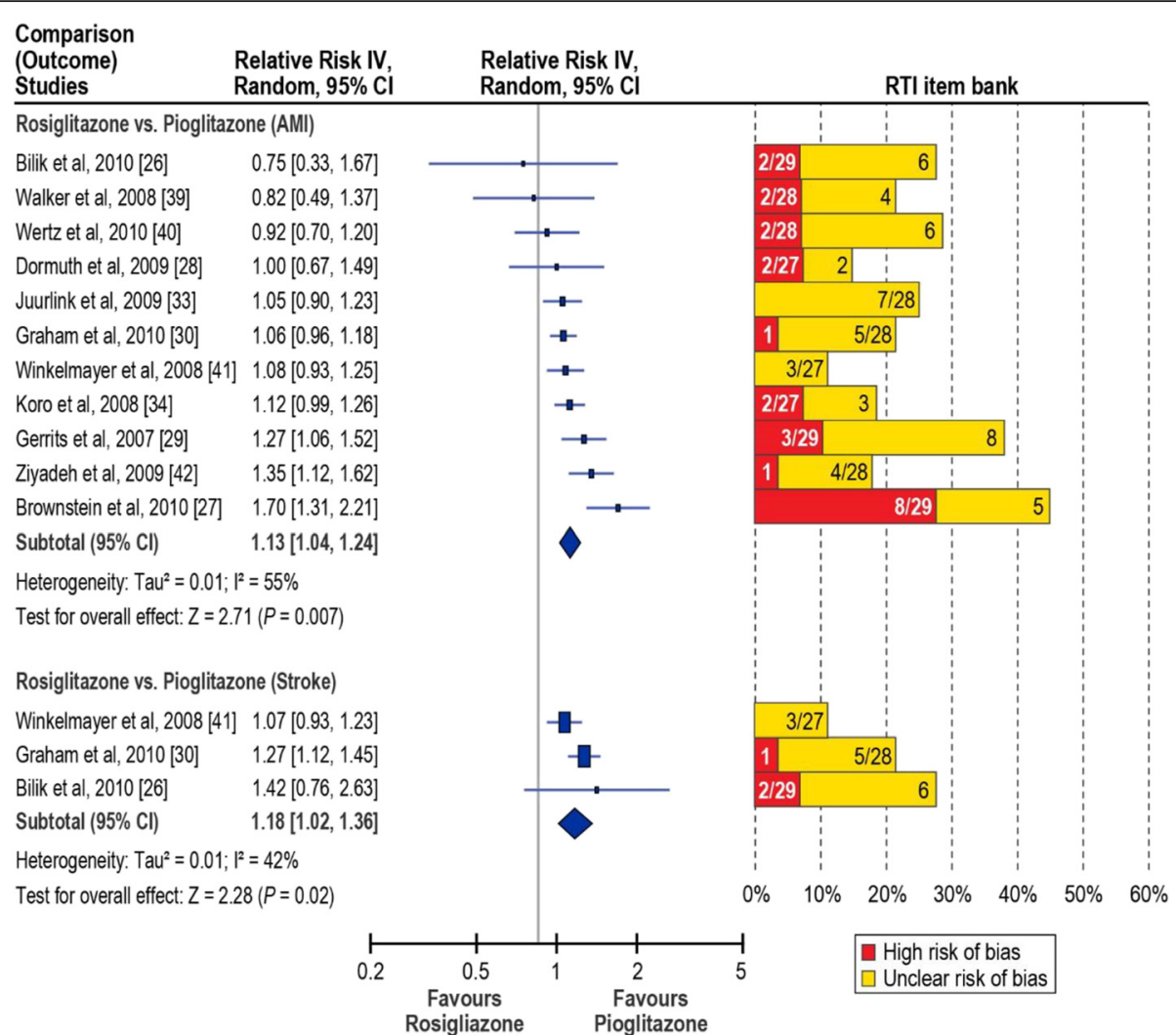

Fig. 2 Relative risk of acute myocardial infarction and stroke in rosiglitazone users compared with pioglitazone users. AMl, acute myocardial infarction; IV, inverse variance. Red bars, percentage of items in the RTI item bank indicating high risk of bias; yellow bars, percentage of items at unclear risk of bias. Items with low risk of bias not shown. Denominators indicate the number of items evaluated for each study.

3 cohort studies of combination therapy (Fig. 2). Two studies used Medicare data and studied patients aged 65 years and older; 1 included patients aged 18 years or older. None reported information on dose or duration.

\section{Risk of AMI: rosiglitazone versus metformin}

The overall sRR (95\% CI) was 1.42 (1.03-1.98) (Fig. 3), but, there was strong evidence of heterogeneity $\left(I^{2}=86 \%\right)$. With the RTI-IB quality assessment, 2 studies had a high/ unclear risk of bias for $30 \%$ or more of the items assessed [27, 32]; and 1 more study had a high/unclear risk of bias for $25 \%$ or more of the items assessed [36].

Subgroup analyses showed that heterogeneity was reduced when restricting the analysis to new users of rosiglitazone and metformin $\left(I^{2}=55 \%\right)$ (Table 4). Even larger reductions in heterogeneity were obtained in the sensitivity analyses excluding the two studies at high/unclear risk of bias for $30 \%$ or more of the items assessed $\left(I^{2}=0 \%\right)$. The sRR decreased in the subgroup analysis (new users) to 1.29 and to 1.13 in the overall sensitivity analysis; the null effect was included in the subgroup analyses random-effects $95 \% \mathrm{CI}$ and the overall sensitivity analysis random- and fixed-effects $95 \%$ CIs.
All 7 studies included in the meta-analysis used a cohort design, but 1 study performed a nested case-control analysis. Only 1 study was restricted to first-ever events. Of the 7 studies, 5 included only new users of the drugs studied, 4 reported estimates only for monotherapy drug use, 1 reported estimates only of comparisons in which the rosiglitazone and pioglitazone groups allowed combination treatment with other oral blood glucose-lowering drugs, and 2 provided estimates for both monotherapy and combination therapy. Only 1 study provided results by exposure duration.

\section{Risk of AMI: pioglitazone versus metformin}

The sRR $(95 \% \mathrm{CI})$ was $1.02(0.75-1.38)$, and there was no evidence of significant heterogeneity in a metaanalysis of 3 studies $\left(I^{2}=17 \%\right)$. Four studies were available for this comparison (Fig. 3); however, the $95 \%$ CI reported by Dormuth et al. [28] did not result in an estimable standard error and therefore the study could not be included in the meta-analysis.

\section{Risk of AMI: rosiglitazone versus sulfonylureas}

The sRR (95\% CI) was 0.99 (0.78-1.25), with strong evidence of heterogeneity for this comparison $\left(I^{2}=70 \%\right)$ 


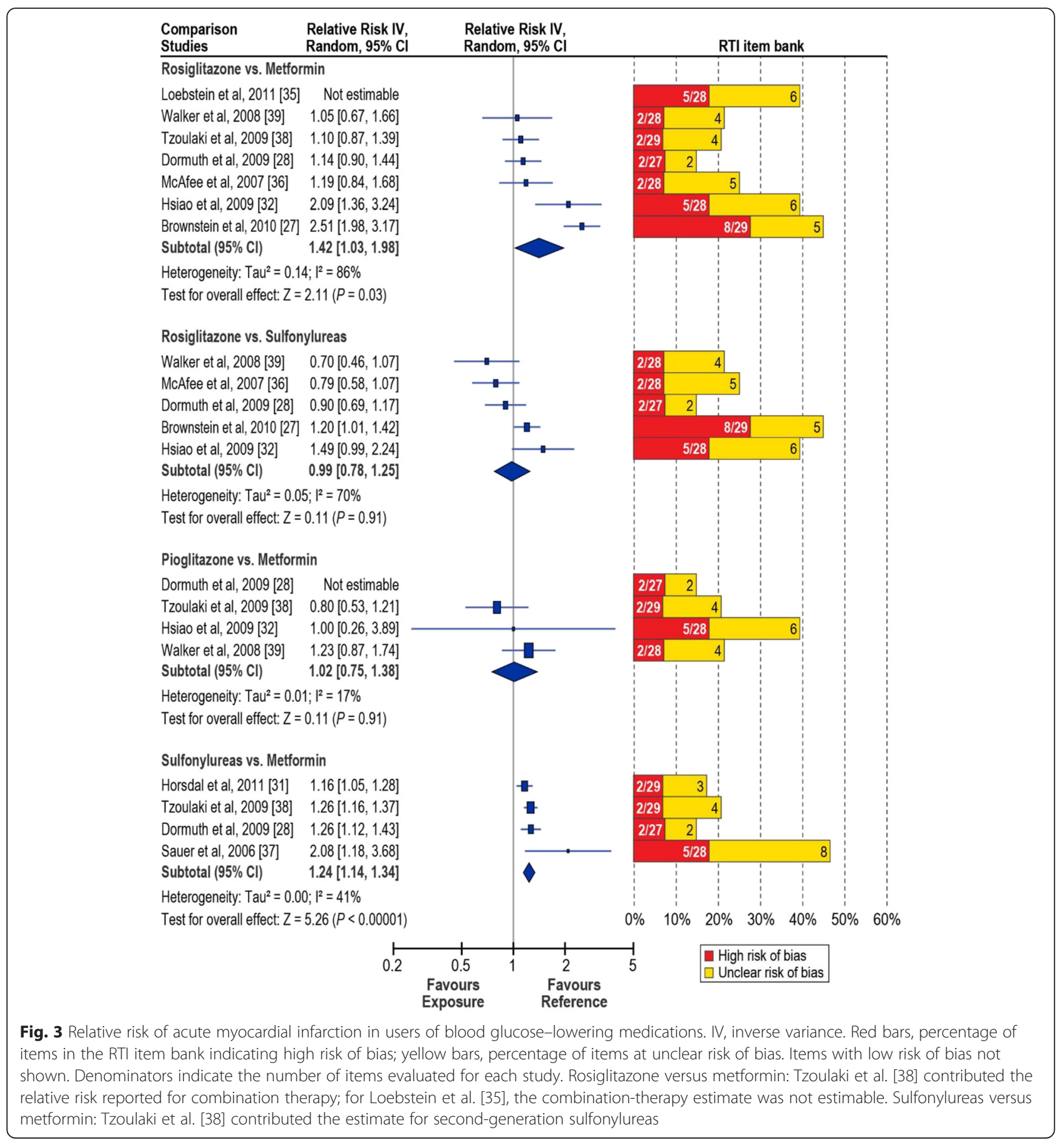

(Fig. 3 and Additional file 1: Table 8e). Five studies were available for meta-analysis. In the sensitivity analysis that excluded the high/unclear-bias studies [27, 32], heterogeneity was markedly reduced $\left(I^{2}=0 \%\right)$ and the sRR (95 $\%$ CI) decreased to $0.82(0.69-0.98)$.

\section{Risk of AMl: sulfonylureas versus metformin}

The sRR (95\% CI) was 1.24 (1.14-1.34) and evidence of heterogeneity was moderate $\left(I^{2}=41 \%\right)$. Four studies were available for this comparison (Fig. 3, Additional file 1: Table 9e); all analysed sulphonylureas as a group and some also reported on individual agents. Individual agents in the evaluated studies were either first- or second-generation sulphonylureas. For our analysis, we included the reported RR for second-generation sulfonylureas as they were the most frequently used. Three of the four studies $[28,31,38]$ provided specific data for the comparison of glyburide versus metformin. When 
Table 3 Risk of AMI in rosiglitazone users compared with pioglitazone users, overall and subgroup analyses

\begin{tabular}{|c|c|c|c|c|c|c|}
\hline \multirow{3}{*}{$\begin{array}{l}\text { Study (author, } \\
\text { year) }\end{array}$} & \multirow[t]{3}{*}{ Overall RR (95 \% Cl) } & \multicolumn{5}{|l|}{ Subgroup analyses } \\
\hline & & \multirow{2}{*}{$\begin{array}{l}\text { Overall sensitivity } \\
\text { analysis }^{a}\end{array}$} & \multicolumn{2}{|c|}{ Incident and prevalent cases } & \multicolumn{2}{|l|}{ New users } \\
\hline & & & & Sensitivity analysis ${ }^{a}$ & & Sensitivity analysis ${ }^{2}$ \\
\hline Walker, 2008 [39] & $0.82(0.49-1.37)$ & & & & & \\
\hline Ziyadeh, 2009 [42] & $1.35(1.12-1.62)$ & & & & & \\
\hline $\begin{array}{l}\text { Brownstein, } 2010 \\
\text { [27] }\end{array}$ & $1.70(1.31-2.21)$ & Not included & & Not included & Not reported & Not reported \\
\hline Bilik, 2010 [26] & $0.75(0.33-1.67)$ & & & & Not reported & Not reported \\
\hline Wertz, 2010 [40] & $0.92(0.70-1.20)$ & & & & & \\
\hline $\begin{array}{l}\text { Dormuth, } 2009 \\
\text { [28] }\end{array}$ & $1.00(0.67-1.49)$ & & & & & \\
\hline Juurlink, 2009 [33] & $1.05(0.90-1.23)$ & & & & & \\
\hline Graham, 2010 [30] & $1.06(0.96-1.18)$ & & & & & \\
\hline $\begin{array}{l}\text { Winkelmayer, } 2008 \\
\text { [41] }\end{array}$ & $1.08(0.93-1.25)$ & & Not reported & & & \\
\hline Koro, 2008 [34] & $1.12(0.99-1.26)$ & & Not reported & & Not reported & Not reported \\
\hline Gerrits, 2007 [29] & $1.27(1.06-1.52)$ & Not included & & Not included & & Not included \\
\hline $\begin{array}{l}\text { Fixed-effects, sRR } \\
(95 \% \mathrm{Cl})\end{array}$ & $1.12(1.06-1.18)$ & $1.09(1.03-1.15)$ & $1.13(1.06-1.21)$ & $1.08(1.00-1.16)$ & $1.10(1.03-1.17)$ & $1.08(1.01-1.15)$ \\
\hline $\begin{array}{l}\text { Random-effects, } \\
\text { sRR ( } 95 \% \mathrm{Cl})\end{array}$ & $1.13(1.04-1.24)$ & $1.09(1.02-1.16)$ & $1.14(1.00-1.30)$ & $1.07(0.96-1.19)$ & $1.10(1.01-1.20)$ & $1.08(0.99-1.18)$ \\
\hline $\begin{array}{l}\text { Heterogeneity } \\
\text { statistics }\end{array}$ & $\begin{array}{l}T 2=0.01 \times 2=22.05 \\
\mathrm{df}=10(P=0.01) P^{2}= \\
55 \%\end{array}$ & $\begin{array}{l}T 2=0.00 \times 2=9.56 \\
\mathrm{df}=8(P=0.30) P^{2}= \\
16 \%\end{array}$ & $\begin{array}{l}T 2=0.02 \times 2=21.74 \\
\mathrm{df}=8(P=0.005) P^{2}= \\
63 \%\end{array}$ & $\begin{array}{l}\tau 2=0.01 \times 2=9.26 \\
\mathrm{df}=6(P=0.16) P^{2}= \\
35 \%\end{array}$ & $\begin{array}{l}\tau 2=0.01 \times 2=11.06 \\
\mathrm{df}=7(P=0.14) P^{2}= \\
37 \%\end{array}$ & $\begin{array}{l}\tau 2=0.00 \times 2=8.47 \\
d f=6(P=0.21) P^{2}= \\
29 \%\end{array}$ \\
\hline
\end{tabular}

Not reported indicates that the study did not provide an estimate for that subgroup analysis. Not included indicates that the study was removed as part of the sensitivity analysis

$d f$ degrees of freedom, $R R$ relative risk, sRR summary relative risk

${ }^{a}$ Sensitivity analysis: we excluded those studies with a combined high or unclear risk of bias for more than $30 \%$ of the items in the RTI item bank

Table 4 Risk of AMI in rosiglitazone users compared with metformin users, overall and subgroup analyses

\begin{tabular}{|c|c|c|c|c|}
\hline \multirow[t]{3}{*}{ Study (author, year) } & \multirow[t]{3}{*}{ Overall RR (95 \% Cl) } & \multirow{3}{*}{ Overall sensitivity analysis $^{a}$} & \multicolumn{2}{|l|}{ Subgroup analyses } \\
\hline & & & \multicolumn{2}{|l|}{ New users } \\
\hline & & & & Sensitivity analysis ${ }^{a}$ \\
\hline Loebstein, 2011 [35] & Not estimable & Not estimable & Not estimable & Not estimable \\
\hline Walker, 2008 [39] & $1.05(0.67-1.66)$ & & & \\
\hline Tzoulaki, 2009 [38] & $1.10(0.87-1.39)$ & & Not reported & Not reported \\
\hline Dormuth, 2009 [28] & $1.14(0.90-1.44)$ & & & \\
\hline McAfee, 2007 [36] & $1.19(0.84-1.68)$ & & & \\
\hline Hsiao, 2009 [32] & $2.09(1.36-3.24)$ & Not Included & & Not included \\
\hline Brownstein, 2010 [27] & $2.51(1.98-3.17)$ & Not Included & Not reported & Not reported \\
\hline Fixed-effects, sRR (95\% Cl) & $1.44(1.28-1.61)$ & $1.13(0.98-1.30)$ & $1.24(1.05-1.47)$ & $1.14(0.95-1.36)$ \\
\hline Random-effects, sRR (95\% Cl) & $1.42(1.03-1.98)$ & $1.13(0.98-1.30)$ & $1.29(0.99-1.67)$ & $1.14(0.95-1.36)$ \\
\hline Hetrogeneity statistics & $\begin{array}{l}T^{2}=0.14 x^{2}=36.07, \mathrm{df}=5 \\
(P<0.00001) P^{2}=86 \%\end{array}$ & $\begin{array}{l}T^{2}=0.00 x^{2}=0.21, d f=3 \\
(P=0.98) P^{2}=0 \%\end{array}$ & $\begin{array}{l}\tau^{2}=0.04 X^{2}=6.62, \mathrm{df}=3 ; \\
\left.(P=0.09)\right|^{2}=55 \%\end{array}$ & $\begin{array}{l}T^{2}=0.00 x^{2}=0.17, \mathrm{df}=2 ; \\
(P=0.92) P^{2}=0 \%\end{array}$ \\
\hline
\end{tabular}


combining those studies (Additional file 1: Table 10e), effect estimates were homogeneous across studies and there was no evidence of heterogeneity $\left(I^{2}=0 \%\right)$. The sRR (95\% CI) was 1.22 (1.14-1.31). Two of the four studies were included in the meta-analysis for only this comparison [31, 37]. One was a field case-control study with an imprecise RR estimate and $30 \%$ or more of the RTI-IB items assessed at high/unclear risk of bias. The other was a nested case-control analysis that included prevalent and new users. Three studies included only first-ever AMI.

No impact on the meta-analysis results was found after grouping the studies according to whether the study period finished before or on 2007, started after 2007, or the study period included 2007 (data not shown).

\section{Publication bias}

Examination of funnel plots did not suggest publication bias, although the number of studies was small for some comparisons. The funnel plots for studies evaluating the risk of AMI in rosiglitazone users compared with pioglitazone users $(n=11)$, in rosiglitazone users compared with metformin users $(n=7)$, and in rosiglitazone users compared with sulfonylurea users $(n=5)$ are displayed in Additional file 1: Figures 1e, 2e, and 3e.

\section{Discussion}

This systematic review of published observational studies on the risk of AMI associated with glitazones, metformin and sufonylureas in patients with T2DM confirmed that studies in this field are very heterogeneous in exposure definition, comparison drugs, potential for biases secondary to design characteristics, and study results. Therefore, summarising the scientific evidence is challenging. The lack of a common reference medication for evaluation of all potential exposures across studies limited direct comparison of effect estimates. Of the 31 studies included in our systematic literature review, only 20 could contribute to the metaanalysis, 17 for AMI and 3 for stroke.

Our summary effect estimates are compatible with a small increase (around 10\%) in the risk of AMI among patients with T2DM using rosiglitazone compared with the risk in those using pioglitazone. However, the degree of heterogeneity present and results of the quality assessment and sensitivity analyses indicate that caution should be used when interpreting this result. Residual confounding might be present in most observational studies; therefore, small increases in risk are difficult to interpret.

Studies in the stroke risk meta-analysis $(n=3)$ had more homogeneous point estimates and quality assessments than studies in the AMI meta-analysis $(n=11)$.
Considering that the lower limit of the $95 \% \mathrm{CI}$ is very close to 1 and only 3 studies were available for metaanalysis, the observed increase of $18 \%$ in the risk of stroke among patients using rosiglitazone compared with those using pioglitazone should be considered cautiously.

Similar words of caution apply to the comparisons of rosiglitazone versus metformin and rosiglitazone versus sulfonylureas. Although the sRRs indicate a $40 \%$ increase in risk of AMI for the first comparison and no increase in risk for the second comparison, there was strong evidence of heterogeneity for both comparisons, and the sensitivity analyses produced effect estimates closer to the null for the first comparison and further from the null for the second comparison (from sRR of 0.99 to 0.92 in the subgroup analysis and to 0.82 in the sensitivity analysis).

Results reported from the individual studies included in other pioglitazone-versus-metformin and sulfonylureasversus-metformin comparisons produced more homogeneous results but involved few studies. The sRR for pioglitazone versus metformin suggested no difference in AMI risk for the two drugs, but the other comparison indicated a $24 \%$ higher risk of AMI for sulfonylureas users than for metformin users.

In September 2014, we updated the literature search using the original search terms in PubMed. Two new studies were considered eligible and were reviewed $[43,44]$; they compared risk of AMI and of stroke for rosiglitazone and pioglitazone. The updated effect estimates adding these 2 studies remained virtually unchanged: sRR, 1.12 (95 \% CI, 1.03-1.21) for AMI and 1.17 (95\% CI, 1.07, 1.27) for stroke.

The results of our meta-analysis are consistent with results of two previous meta-analyses of observational studies evaluating the risk of AMI comparing rosiglitazone and pioglitazone. The meta-analysis of 7 studies conducted by Chen et al. [16] estimated a sRR of 1.17 (95\% CI, 1.04-1.32); $I^{2}=70 \%$. Loke et al. [11] conducted a meta-analysis of 13 studies including patients with T2DM, comparing rosiglitazone and pioglitazone safety for AMI, congestive heart failure, and mortality. For AMI, the odds ratio was 1.11 (95\% CI, 1.04-1.18) ; $I^{2}=16 \%$. These estimated relative risks are similar to our estimate. Differences in the studies included/excluded between those two meta-analyses and our meta-analysis might explain the differences in statistical heterogeneity.

Three meta-analyses of randomised controlled trials (RCTs) have been published evaluating the safety of rosiglitazone for AMI and stroke and found that rosiglitazone increased the risk of AMI about $40 \%$ compared to control therapies or placebo [12-14]. The results of those meta-analysis were not without controversy [45], and there is evidence that they are sensitive to the 
statistical method used for pooling and the inclusion/exclusion of different studies [46]. However, the available evidence prompted the European Medicines Agency to withdraw rosiglitazone from the market and the FDA to place severe restrictions on rosiglitazone use [5]. In contrast, meta-analyses indicate a reduced risk of stroke (about $20 \%$ ) for rosiglitazone compared to control therapies. Although the $95 \%$ CIs of those estimates include the null effect, the inconsistency of the findings for AMI and stroke is difficult to explain [46]. An independent re-analysis of the RECORD trial data confirmed the original results [9], that rosiglitazone did not increase cardiovascular risk compared to a combination of metformin and sulfonylurea [47]; this has prompted the FDA to ease some restrictions on rosiglitazone use. Another re-analysis of clinical trial data has further questioned the adverse cardiovascular safety profile of rosiglitazone, reporting a hazard ratio of 0.77 for AMI (95\% CI, 0.54-1.10) and of 0.36 (95\% CI, 0.16-0.86) for stroke when comparing rosiglitazone with nonthiazolidinedione use [7].

We did not identify any meta-analysis of RCTs directly comparing rosiglitazone and pioglitazone with AMI as the safety outcome of interest. Two meta-analyses of RCTs comparing pioglitazone with control therapies or placebo found reductions of around $20 \%$ in the risk of AMI or stroke, favouring pioglitazone, with $95 \%$ CIs in both cases including the null effect $[48,49]$.

For AMI and stroke we could not identify any published meta-analyses of observational studies evaluating the other drug comparisons included in this meta-analysis, i.e., rosiglitazone or pioglitazone versus metformin, rosiglitazone versus sulfonylureas, and sulfonylureas versus metformin. Therefore, the current meta-analysis seems to be the first one to review the results of studies including all those comparisons.

This systematic review and meta-analysis has several strengths. We included in the meta-analysis only studies with a clear definition of the reported comparisons. Our detailed evaluation of the quality of each reviewed study and the sensitivity analyses helped us interpret metaanalysis results of heterogeneous studies combined for the purpose of evaluating the risk of AMI and stroke. Our analyses included drug comparisons relevant to clinicians and not included in previous meta-analyses.

As is true for every meta-analysis of observational studies, the main limitation of this meta-analysis is the heterogeneity in design and conduct of the primary studies. Key drivers of this heterogeneity were the complex array of treatment options, varying severity of diabetes, and varying outcome definitions. The studies that combined medications (e.g., "any other treatment") as the reference group are of particular concern for this and future meta-analyses for two main reasons. First, "any other treatment" represents different treatments depending on the study period and population, which decreases the applicability and comparability of results. Second, results relative to such reference treatment may not be useful for clinical decision makers who need to choose between specific therapeutic alternatives. The results of this study show that methodological limitations present in the original studies have an important role in explaining the statistical heterogeneity found when combining the individual study results in the meta-analysis. When excluding those studies at high/unclear risk of bias for $30 \%$ or more of the items assessed by the RTIIB, heterogeneity was largely reduced in most of the comparisons analysed. Overall results in those comparisons were sensitive to the exclusion of the studies more prone to bias, which indicates lack of robustness in results when including such studies in the meta-analysis.

Few of the studies included accounted for severity of diabetes; therefore, confounding by indication could be present in the majority of within-study comparisons for studies in this meta-analysis. Residual confounding might be present in studies that failed to systematically record or adjust for lifestyle factors. Few studies adjusted for socioeconomic status, education, physical activity, or BMI, which can all be associated with both treatment selection and the development of outcomes. These and other methodological limitations of the majority of the studies included in this meta-analysis have been reviewed in recent publications, which support the overall qualitative findings of this study [50, 51]. Since the magnitude of the increased risks was rather small for most of the comparisons, small residual confounding, if present, could explain the small increases in the risk estimates.

Evaluation of the risk of stroke was limited due to the small number of published studies and inclusion of only users of thiazolidinediones. Other limitations of this meta-analysis had to do with the fact that dose and duration effects could not be evaluated for any comparison due to the scarcity of data in the published studies and the fact that the newest oral drugs for diabetes could not be evaluated due to lack of studies.

\section{Conclusions}

In conclusion, sulfonylureas seem to increase the risk of AMI by $24 \%$ compared with metformin. With 3 small studies and a corresponding lack of precision, results of this meta-analysis suggest no difference in the risk of AMI for pioglitazone compared with metformin. The presence of heterogeneity in the meta-analysis results precludes any conclusions on the risk of AMI for the other comparisons evaluated. The quality assessment with the RTI-IB and the sensitivity analyses indicate that statistical heterogeneity might be attributable to the 
studies that were at the highest risk of bias according to the RTI-IB. Future studies should consider the methodological pitfalls identified in the existing body of evidence. Results from ongoing large multidatabase studies, carefully planned and conducted, are awaited and will help to elucidate the risk of AMI and stroke associated with the use of NIBGLDs.

\section{Competing interests}

RTI Health Solutions employees work on projects funded by pharmaceutical companies including manufacturers of treatments for patients with diabetes. As employees of RTI Health Solutions, Manel Pladevall, Susana Perez-Gutthann, and Cristina Varas-Lorenzo also participate in advisory boards funded by pharmaceutical companies. The authors declare that they have no competing interests

\section{Authors' contribution}

MP, NRG, CVL, AM, and SPG participated in development of the literature search strategy; MP, NRG, and AM abstracted and compiled the data; NRG, BC, AM, MP, and CVL performed the analyses; all authors oversaw design of the study and facilitated interpretation of the findings. MP drafted the manuscript; all coauthors reviewed and revised it critically for important intellectual content and read and approved the final manuscript. All authors had full access to all of the data abstracted from published studies included in this systematic review and take responsibility for the integrity of summarising the data and the accuracy of the meta-analysis.All authors read and approved the final manuscript

\section{Additional file}

Additional file 1: Table 1e. Search Terms for Medline search. Table 2e. Newcastle-Ottawa Scale Quality Assessment Results, Individual CaseControl Studies Assessing the Risk of Acute Myocardial Infarction. Table 3e. Newcastle-Ottawa Scale Quality Assessment Results, Individual Cohort Studies Assessing the Risk of Acute Myocardial Infarction. Table 4e. Newcastle-Ottawa Scale Quality Assessment Results, Individual CaseControl Studies Assessing the Risk of Stroke. Table 5e. Newcastle-Ottawa Scale Quality Assessment Results, Individual Cohort Studies Assessing the Risk of Stroke. Table 6e. RTI Item Bank Quality Assessment Results: Individual Studies Reporting on the Risk of Acute Myocardial Infarction. Table 7e. RTI Item Bank Quality Assessment Results: Individual Studies Reporting on the Risk of Stroke. Table 8e. Risk of Acute Myocardial Infarction in Rosiglitazone Users Compared with the Risk in Sulfonylurea Users: Overall, Subgroup (New Users), and Sensitivity Analysis. Table 9e. Risk of Acute Myocardial Infarction in Sulfonylurea Users Compared with the Risk in Metformin Users: Overall, and in a Sensitivity Analysis - Summary Relative Risk by Random Effects. Table 10e. Risk of Acute Myocardial Infarction in Glyburide Users Compared with the Risk in Metformin Users: Overall Summary Relative Risk by Random Effects. Figure 1e. Funnel Plot of the Relative Risk of Acute Myocardial Infarction for Rosiglitazone Users Compared With Pioglitazone Users (11 Studies). Figure 2e. Funnel Plot of the Relative Risk of Acute Myocardial Infarction for Rosiglitazone Users Compared With Metformin Users (7 Studies). Figure 3e. Funnel Plot of the Relative Risk of Acute Myocardial Infarction for Rosiglitazone Users Compared With Sulfonylureas Users (5 Studies). (DOCX 278 kb)

\section{Abbreviations}

AMI: Acute myocardial infarction; ESM: Electronic supplementary material; FDA: US Food and Drug Administration; P $^{2}$ : Heterogeneity statistic, interpreted as the percentage of variability on a set of effect size estimates due to heterogeneity between studies rather than sampling error; MOOSE: Meta-analysis of observational studies in epidemiology; NIBGLD: Non-insulin blood glucose-lowering drugs; RCT: Randomised controlled trial; RTI-IB: RTI item bank; sRR: Summary relative risk; T2DM: Type 2 diabetes mellitus; US: United States of America.

\section{Acknowledgements}

We would like to thank Lorenza Scotti for developing the Microsoft Access database for collecting abstracted data; Mark Howell, Information Services Specialist, for his support and contributions in developing the literature search strategy; Adele C. Monroe, Medical Editor, for her expert editorial support; Candace Webster, Graphic Artist, for her work on the figures in this manuscript; and Carla Franzoni for her support in managing the project.

\section{Funding}

This research has received funding from the European Community's Seventh Framework Programme (FP7/2007-2013) under grant agreement number 282521 - the SAFEGUARD project [60].

\section{Author details}

${ }^{1}$ RTI Health Solutions, Trav. Gracia 56 Atico 1 08006, Barcelona, Spain. ${ }^{2}$ The Center for Health Policy and Health Services Research, Henry Ford Health System, Detroit, Michigan, USA. ${ }^{3}$ RTI Health Solutions, Research Triangle Park, NC, USA.

Received: 3 September 2015 Accepted: 8 January 2016

Published online: 15 January 2016

\section{References}

1. Whiting DR, Guariguata L, Weil C, Shaw J. IDF diabetes atlas: global estimates of the prevalence of diabetes for 2011 and 2030. Diabetes Res Clin Pract. 2011;94(3):311-21.

2. Perk J, De Backer G, Gohlke H, Graham I, Reiner Z, Verschuren M, et al European Guidelines on cardiovascular disease prevention in clinical practice (version 2012): The Fifth Joint Task Force of the European Society of Cardiology and Other Societies on Cardiovascular Disease Prevention in Clinical Practice (constituted by representatives of nine societies and by invited experts) * Developed with the special contribution of the European Association for Cardiovascular Prevention \& Rehabilitation (EACPR). Eur Heart J. 2012;33(13):1635-701.

3. Bulugahapitiya U, Siyambalapitiya S, Sithole J, Idris I. Is diabetes a coronary risk equivalent? Systematic review and meta-analysis. Diabet Med. 2009; 26(2):142-8.

4. Lee C, Joseph L, Colosimo A, Dasgupta K. Mortality in diabetes compared with previous cardiovascular disease: a gender-specific meta-analysis. Diabetes Metab. 2012;38(5):420-7.

5. Nissen SE. Cardiovascular effects of diabetes drugs: emerging from the dark ages. Ann Intern Med. 2012;157(9):671-2.

6. Gerstein HC, Miller ME, Byington RP, Goff Jr DC, Bigger JT, Buse JB, et al. Effects of intensive glucose lowering in type 2 diabetes. N Engl J Med. 2008; 358(24):2545-59

7. Bach RG, Brooks MM, Lombardero M, Genuth S, Donner TW, Garber A, et al. Rosiglitazone and outcomes for patients with diabetes mellitus and coronary artery disease in the bypass angioplasty revascularization investigation 2 diabetes (BARI 2D) trial. Circulation. 2013;128(8):785-94.

8. Gerstein HC. Rosiglitazone and cardiovascular outcomes: is there a clear answer? Circulation. 2013:128(8):777-9.

9. Hiatt WR, Kaul S, Smith RJ. The cardiovascular safety of diabetes drugs -insights from the rosiglitazone experience. N Engl J Med. 2013; 369(14):1285-7.

10. Roumie CL, Hung AM, Greevy RA, Grijalva CG, Liu X, Murff HJ, et al. Comparative effectiveness of sulfonylurea and metformin monotherapy on 
cardiovascular events in type 2 diabetes mellitus: a cohort study. Ann Intern Med. 2012;157(9):601-10.

11. Loke YK, Kwok CS, Singh S. Comparative cardiovascular effects of thiazolidinediones: systematic review and meta-analysis of observational studies. BMJ. 2011;342:d1309.

12. Singh $\mathrm{S}$, Loke $Y K$, Furberg $C D$. Long-term risk of cardiovascular events with rosiglitazone: a meta-analysis. JAMA. 2007;298(10):1189-95.

13. Nissen SE, Wolski K. Effect of rosiglitazone on the risk of myocardial infarction and death from cardiovascular causes. N Engl J Med. 2007;356(24):2457-71.

14. Cobitz A, Zambanini A, Sowell M, Heise M, Louridas B, McMorn S, et al. A retrospective evaluation of congestive heart failure and myocardial ischemia events in 14,237 patients with type 2 diabetes mellitus enrolled in 42 shortterm, double-blind, randomized clinical studies with rosiglitazone. Pharmacoepidemiol Drug Saf. 2008;17(8):769-81.

15. Phung OJ, Schwartzman E, Allen RW, Engel SS, Rajpathak SN. Sulphonylureas and risk of cardiovascular disease: systematic review and meta-analysis. Diabet Med. 2013:30(10):1160-71.

16. Chen X, Yang L, Zhai SD. Risk of cardiovascular disease and all-cause mortality among diabetic patients prescribed rosiglitazone or pioglitazone: a meta-analysis of retrospective cohort studies. Chin Med J (Engl). 2012; 125(23):4301-6.

17. Mannucci E, Monami M, Di Bari M, Lamanna C, Gori F, Gensini GF, et al. Cardiac safety profile of rosiglitazone: a comprehensive meta-analysis of randomized clinical trials. Int J Cardiol. 2010;143(2):135-40.

18. Monami M, Genovese S, Mannucci E. Cardiovascular safety of sulfonylureas: a meta-analysis of randomized clinical trials. Diabetes Obes Metab. 2013; 15(10):938-53.

19. Berlin JA, Colditz GA. The role of meta-analysis in the regulatory process for foods, drugs, and devices. JAMA. 1999;281(9):830-4.

20. Varas-Lorenzo C, Margulis AV, Pladevall M, Riera-Guardia N, Calingaert B, Hazell $L$, et al. The risk of heart failure associated with the use of noninsulin blood glucose-lowering drugs: systematic review and meta-analysis of published observational studies. BMC Cardiovasc Disord. 2014;14(1):129.

21. Wells G, Shea B, O'Connell D, Peterson J, Welch V, Losos M, Tugwell P: The Newcastle-Ottawa Scale (NOS) for assessing the quality of nonrandomised studies in meta-analyses. 2011

22. Viswanathan $M$, Berkman ND. Development of the RTI item bank on risk of bias and precision of observational studies. J Clin Epidemiol. 2012;65(2):163-78.

23. Margulis AV, Pladevall M, Riera-Guardia N, Varas-Lorenzo C, Hazell L, Berkman ND, et al. Quality assessment of observational studies in a drugsafety systematic review, comparison of two tools: the Newcastle-Ottawa Scale and the RTI item bank. Clin Epidemiol. 2014;6:359-68.

24. The Cochrane Collaboration. Review Manager (RevMan) [Computer Program] Version 5.2.3. Copenhagen: The Nordic Cochrane Centre; 2012. http://tech.cochrane.org/revman. Accessed 12 December 2014.

25. Stroup DF, Berlin JA, Morton SC, Olkin I, Williamson GD, Rennie D, et al. Meta-analysis of observational studies in epidemiology: a proposal for reporting. Meta-analysis of observational studies in epidemiology (MOOSE) group. JAMA. 2000;283(15):2008-12

26. Bilik D, McEwen LN, Brown MB, Selby JV, Karter AJ, Marrero DG, et al. Thiazolidinediones, cardiovascular disease and cardiovascular mortality: translating research into action for diabetes (TRIAD). Pharmacoepidemiol Drug Saf. 2010;19(7):715-21.

27. Brownstein JS, Murphy SN, Goldfine AB, Grant RW, Sordo M, Gainer V, et al. Rapid identification of myocardial infarction risk associated with diabetes medications using electronic medical records. Diabetes Care. 2010;33(3):526-31.

28. Dormuth CR, Maclure M, Carney G, Schneeweiss S, Bassett K, Wright JM. Rosiglitazone and myocardial infarction in patients previously prescribed metformin. PLoS One. 2009;4(6), e6080.

29. Gerrits CM, Bhattacharya M, Manthena S, Baran R, Perez A, Kupfer S. A comparison of pioglitazone and rosiglitazone for hospitalization for acute myocardial infarction in type 2 diabetes. Pharmacoepidemiol Drug Saf. 2007:16(10):1065-71.

30. Graham DJ, Ouellet-Hellstrom R, MaCurdy TE, Ali F, Sholley C, Worrall C, et al. Risk of acute myocardial infarction, stroke, heart failure, and death in elderly Medicare patients treated with rosiglitazone or pioglitazone. JAMA. 2010;304(4):411-8

31. Horsdal HT, Sondergaard F, Johnsen SP, Rungby J. Antidiabetic treatments and risk of hospitalisation with myocardial infarction: a nationwide casecontrol study. Pharmacoepidemiol Drug Saf. 2011;20(4):331-7.
32. Hsiao FY, Huang WF, Wen YW, Chen PF, Kuo KN, Tsai YW. Thiazolidinediones and cardiovascular events in patients with type 2 diabetes mellitus: a retrospective cohort study of over 473,000 patients using the National Health Insurance database in Taiwan. Drug Saf. 2009;32(8):675-90.

33. Juurlink DN, Gomes T, Lipscombe LL, Austin PC, Hux JE, Mamdani MM. Adverse cardiovascular events during treatment with pioglitazone and rosiglitazone: population based cohort study. BMJ. 2009;339:b2942.

34. Koro CE, Fu Q, Stender M. An assessment of the effect of thiazolidinedione exposure on the risk of myocardial infarction in type 2 diabetic patients. Pharmacoepidemiol Drug Saf. 2008;17(10):989-96.

35. Loebstein R, Dushinat M, Vesterman-Landes J, Silverman B, Friedman N, Katzir I, et al. Database evaluation of the effects of long-term rosiglitazone treatment on cardiovascular outcomes in patients with type 2 diabetes. J Clin Pharmacol. 2011;51(2):173-80.

36. McAfee AT, Koro C, Landon J, Ziyadeh N, Walker AM. Coronary heart disease outcomes in patients receiving antidiabetic agents. Pharmacoepidemiol Drug Saf. 2007:16(7):711-25.

37. Sauer WH, Cappola AR, Berlin JA, Kimmel SE. Insulin sensitizing pharmacotherapy for prevention of myocardial infarction in patients with diabetes mellitus. Am J Cardiol. 2006;97(5):651-4.

38. Tzoulaki I, Molokhia M, Curcin V, Little MP, Millett CJ, Ng A, et al. Risk of cardiovascular disease and all cause mortality among patients with type 2 diabetes prescribed oral antidiabetes drugs: retrospective cohort study using UK general practice research database. BMJ. 2009;339:b4731.

39. Walker AM, Koro CE, Landon J. Coronary heart disease outcomes in patients receiving antidiabetic agents in the PharMetrics database 2000-2007. Pharmacoepidemiol Drug Saf. 2008;17(8):760-8.

40. Wertz DA, Chang CL, Sarawate CA, Willey VJ, Cziraky MJ, Bohn RL. Risk of cardiovascular events and all-cause mortality in patients treated with thiazolidinediones in a managed-care population. Circ Cardiovasc Qual Outcomes. 2010;3(5):538-45.

41. Winkelmayer WC, Setoguchi S, Levin R, Solomon DH. Comparison of cardiovascular outcomes in elderly patients with diabetes who initiated rosiglitazone vs pioglitazone therapy. Arch Intern Med. 2008;168(21):2368-75.

42. Ziyadeh N, McAfee AT, Koro C, Landon J, Arnold Chan K. The thiazolidinediones rosiglitazone and pioglitazone and the risk of coronary heart disease: a retrospective cohort study using a US health insurance database. Clin Ther. 2009:31(11):2665-77.

43. Tannen R, Xie D, Wang X, Yu M, Weiner MG. A new "Comparative Effectiveness" assessment strategy using the THIN database: comparison of the cardiac complications of pioglitazone and rosiglitazone. Pharmacoepidemiol Drug Saf. 2013;22(1):86-97.

44. Gallagher AM, Smeeth L, Seabroke S, Leufkens HG, van Staa TP. Risk of death and cardiovascular outcomes with thiazolidinediones: a study with the general practice research database and secondary care data. PLoS One. 2011;6(12), e28157.

45. Diamond GA, Bax L, Kaul S. Uncertain effects of rosiglitazone on the risk for myocardial infarction and cardiovascular death. Ann Intern Med. 2007; 147(8):578-81.

46. Friedrich JO, Beyene J, Adhikari NK. Rosiglitazone: can meta-analysis accurately estimate excess cardiovascular risk given the available data? Reanalysis of randomized trials using various methodologic approaches. BMC Res Notes. 2009:2:5.

47. Home PD, Pocock SJ, Beck-Nielsen H, Curtis PS, Gomis R, Hanefeld M, et al. Rosiglitazone evaluated for cardiovascular outcomes in oral agent combination therapy for type 2 diabetes (RECORD): a multicentre, randomised, open-label trial. Lancet. 2009;373(9681):2125-35.

48. Lincoff AM, Wolski K, Nicholls SJ, Nissen SE. Pioglitazone and risk of cardiovascular events in patients with type 2 diabetes mellitus: a metaanalysis of randomized trials. JAMA. 2007;298(10):1180-8.

49. Nagajothi N, Adigopula S, Balamuthusamy S, Velazquez-Cecena JL, Raghunathan K, Khraisat A, et al. Pioglitazone and the risk of myocardial infarction and other major adverse cardiac events: a meta-analysis of randomized, controlled trials. Am J Ther. 2008;15(6):506-11.

50. Rawson NS. Review of the quality of observational studies of the association between rosiglitazone and acute myocardial infarction. J Popul Ther Clin Pharmacol. 2014;21(2):e214-32.

51. Patorno E, Patrick AR, Garry EM, Schneeweiss S, Gillet VG, Bartels DB, et al. Observational studies of the association between glucose-lowering medications and cardiovascular outcomes: addressing methodological limitations. Diabetologia. 2014;57(11):2237-50. 
52. Chou CC, Chen WL, Kao TW, Chang YW, Loh CH, Wang CC: Incidence of cardiovascular events in which 2 thiazolidinediones are used as add-on treatments for type 2 diabetes mellitus in a Taiwanese population. Clin Ther. 2011; 33(12):1904-13.

53. Dore DD, Trivedi AN, Mor V, Lapane KL: Association between extent of thiazolidinedione exposure and risk of acute myocardial infarction. Pharmacotherapy. 2009; 29(7):775-83.

54. Habib ZA, Tzogias L, Havstad SL, Wells K, Divine G, Lanfear DE, et al: Relationship between thiazolidinedione use and cardiovascular outcomes and all-cause mortality among patients with diabetes: a time-updated propensity analysis. Pharmacoepidemiol Drug Saf. 2009; 18(6):437-47.

55. Horsdal HT, Johnsen SP, Sondergaard F, Jacobsen J, Thomsen RW, Schmitz $\mathrm{O}$, et al:: Sulfonylureas and prognosis after myocardial infarction in patients with diabetes: a population-based follow-up study. Diabetes Metab Res Rev. 2009: 25(6):515-22

56. Horsdal HT, Johnsen SP, Sondergaard F, Rungby J: Type of preadmission glucose-lowering treatment and prognosis among patients hospitalised with myocardial infarction: a nationwide follow-up study. Diabetologia. 2008; 51(4):567-74.

57. Lipscombe LL, Gomes T, Levesque LE, Hux JE, Juurlink DN, Alter DA: Thiazolidinediones and cardiovascular outcomes in older patients with diabetes. JAMA. 2007; 298(22):2634-43.

58. Azoulay L, Schneider-Lindner V, Dell'aniello S, Filion KB, Suissa S: Thiazolidinediones and the risk of incident strokes in patients with type 2 diabetes: a nested case-control study. Pharmacoepidemiol Drug Saf. 2010; 19(4):343-50.

59. Simpson SH, Majumdar SR, Tsuyuki RT, Eurich DT, Johnson JA: Dose-response relation between sulfonylurea drugs and mortality in type 2 diabetes mellitus: a population-based cohort study. CMAJ. 2006; 174(2):169-74.

60. SAFEGUARD: Project website. 2011. http://www.safeguard-diabetes.org/ Accessed 23 Apr 2015

\section{Submit your next manuscript to BioMed Central and we will help you at every step:}

- We accept pre-submission inquiries

- Our selector tool helps you to find the most relevant journal

- We provide round the clock customer support

- Convenient online submission

- Thorough peer review

- Inclusion in PubMed and all major indexing services

- Maximum visibility for your research

Submit your manuscript at www.biomedcentral.com/submit

C Biomed Central 